\title{
Effect of chronic High Intensity Interval Training on glycosylated haemoglobin in people with type 2 diabetes: a meta-analysis
}

review paper

(c) Wroclaw University of Health and Sport Sciences

DOI: https://doi.org/10.5114/hm.2023.107247

\section{MARÍA CRISTINA ARRIETA-LEANDRO, JESSENIA HERNÁNDEZ-ELIZONDO, JUDITH JIMÉNEZ-DÍAZ}

University of Costa Rica, San Pedro Montes de Oca, Costa Rica

\begin{abstract}
Type 2 diabetes is a chronic disease with an increasing prevalence all over the world. The treatment includes an integral intervention with medical treatment, healthy diet, and regular exercise. High Intensity Interval Training has been recently proposed as part of this treatment to improve glycaemic control. The aim of this meta-analysis is to determine the effect of High Intensity Interval Training on glycosylated haemoglobin in people with type 2 diabetes during at least 12 weeks of intervention. The search was performed in EBSCOHost, Web of Science, Scopus, PubMed, and EMBASE. A total of 250 records were obtained and 22 studies met the inclusion criteria to be meta-analysed. The results showed that High Intensity Interval Training could significantly lower glycosylated haemoglobin; in the sedentary control group, glycosylated haemoglobin significantly increased, making glycaemic control worse. The improvement of glycosylated haemoglobin with High Intensity Interval Training can be influenced by diet, intensity of the intervals, and age. There were some limitations because of lack of control group and appropriate information in some studies. An adequate prescription program should be developed by a qualified professional to ensure best results.
\end{abstract}

Key words: High Intensity Interval Training, type 2 diabetes, glycaemic control, HbA1c

\section{Introduction}

Type 2 diabetes (T2D) is a chronic disease whose prevalence has been increasing in the world population during the last years. For example, only in the United States, there are 29.1 million people diagnosed with T2D (9.3\% of the US population) [1]. In Poland, $8 \%$ of the population suffers from diabetes and it is estimated that $25-30 \%$ of adults with diabetes are unaware of their health condition. The prediction shows that diabetes prevalence in Poland will be $11 \%$ by 2040 [2]. In the Costa Rican population, the same trend is observed. Among people aged above 20 years, T2D prevalence equals around 10.5\% [3]. Moreover, according to the Costa Rican Social Security Fund (CCSS, Caja Costarricense de Seguro Social), Costa Rican people lose an average of 13.2 years of their lives because of diabetes [3].
To take care of T2D, a multidisciplinary treatment is necessary, including drugs, healthy diet and lifestyle, and a proper exercise plan [1]. Much research has been performed with reference to this last point, with the main goal of finding the best option of exercising to ensure good glycaemic control [4].

Moreover, glycosylated haemoglobin (HbA1c) is a biochemical measure used for diabetes monitoring. It was recommended for the first time by the World Health Organization in 2006 and it presents a good overview of the glycaemic behaviour of a person during the previous 8-12 weeks [5, 6]. Although the World Health Organization guidelines indicate that HbA1c can be assessed every 8-12 weeks, recent studies recommend an optimal period of 12 weeks [7] because of the red blood cells replacement time [8].

HbA1c is widely used today in the clinical practice; it has also been proposed as a diagnostic criterion in

Correspondence address: María Cristina Arrieta-Leandro, Universidad de Costa Rica, San José, Montes de Oca, Sabanilla, Instalaciones Deportivas de la Universidad de Costa Rica, post code: 11502, Costa Rica, e-mail: mariacris0409@hotmail.com

Received: February 26, 2021

Accepted for publication: June 8, 2021

Citation: Arrieta-Leandro MC, Hernández-Elizondo J, Jiménez-Díaz J. Effect of chronic High Intensity Interval Training on glycosylated haemoglobin in people with type 2 diabetes: a meta-analysis. Hum Mov. 2023;24(1):32-45; doi: https://doi. $\operatorname{org} / 10.5114 / \mathrm{hm} .2023 .107247$. 
addition to control and monitoring. It is relatively easy to assess because it does not require any special physiological condition to be tested (e.g. minimum fasting hours). In people diagnosed with diabetes, a level of HbA1c below $6.5 \%$ is recommended for optimal glycaemic control [5, 6].

According to the American Diabetes Association and the American College of Sports Medicine (ACSM), individuals diagnosed with diabetes should practise moderate to intense aerobic exercise for 150 minutes/ week, complementary with 2-3 sessions of resistance training weekly [9]. Recently, however, an alternative has been proposed, namely High Intensity Interval Training (HIIT). Some studies have compared the effect of aerobic training, resistance training, and HIIT on T2D, and the outcomes bring about different conclusions [10, 11]. Moreover, other studies tested other alternatives like structured walking, finding them potentially beneficial for glycaemic control $[12,13]$.

Today, there are no guidelines or protocols to follow when prescribing HIIT [10, 11]. Some authors suggest a general schedule with big intervals up to 3 minutes of high intensity (beyond $85 \%$ of maximal capacity) with rest periods between 10 seconds and 4 minutes [9]. Another important fact is that there is no standardized physiological parameter to define the maximal capacity or to establish the rest periods between intervals. Because of all this, the recent studies have applied different methodologies and, as a consequence, revealed different outcomes [9-11].

A recently published meta-analysis compared the effect of exercise vs. non-exercise on HbA1c in people with T2D and found beneficial effects of resistance training with an effect size $(E S)$ of $-0.15(-0.31,0.01)$, concurrent training with $E S$ of $-0.67(-1.04,-0.3)$, and aerobic exercise with $E S$ of $-0.77(-1.07,-0.46)$ [14]. Another recent meta-analysis presented a pre-test vs. post-test comparison and noted positive effects of HIIT on HbA1c with ES of $-0.29(-0.55,-0.04)$ when HIIT was compared with a control group with $E S$ of $-0.39(-0.81,0.02)$ in favour of HIIT; in a comparison with moderate intensity continuous training, $E S$ was $-0.37(-0.55,-0.19)$ favouring HIIT. Nevertheless, the study included trials lasting 4 weeks and more [15], so it could have had accuracy problems in HbA1c measurement [7]; also, it did not provide moderator variable analysis [15].

Therefore, the aim of this meta-analysis was to determine the effect of chronic HIIT on HbA1c in people aged above 18 years with T2D after at least 12 weeks of experimental and quasi-experimental intervention. The second goal was to analyse the potential factors that could directly influence this effect.

\section{Material and methods}

\section{Data sources and searches}

This meta-analysis was performed in accordance with the PRISMA statement [16]. In October 2019, the following databases were searched: EBSCOHost (Academic Search Complete, Fuente Académica Premier, MEDLINE, and SportDiscus), Web of Science, Scopus, PubMed, and EMBASE. The Boolean phrase was "(glycated hemoglobin or glycosilated hemoglobin or hemoglobin a1c HbA1c) AND (type 2 diabetes or type 2 diabetes mellitus or t2dm) AND (hit or hiit or high intensity interval training or high intensity training or aerobic interval training or high intensity intermittent training)". The last search update was made in January 2021.

\section{Inclusion criteria}

In accordance with the PICOS criteria, the metaanalysed studies met the following inclusion criteria: the selected participants were subjects aged 18 years or above with a diagnosis of T2D and available to do exercise, pregnant women were excluded $(\mathrm{P})$; the intervention involved the implementation of a HIIT protocol for at least 12 weeks (I); the comparison was between the status before and after intervention (i.e. pre-test vs. post-test) (C); the outcomes were pre-test and post-test $\mathrm{HbA1}$ c measures (O); and the selected studies had an experimental or quasi-experimental design (S).

There was no gender or date of publication limit, and all the records published in Spanish and English were reviewed. Conference abstracts that met the criteria and showed the necessary data to meta-analyse were included, too.

\section{Study selection}

Data selection and codification was performed by one of the authors and then the information was checked by the other 2 authors. All the information was extracted and processed in Microsoft Office Excel ${ }^{\circledR}$. The data involved authors, year of publication, country; information on the participants: age, sex, type of medication, physical activity level, diet; information on the methodology: training method, intervention duration, exercise frequency, duration, and intensity; information about dependent variables to calculate $E S$ : pre-test and post-test HbA1c, standard deviations, and sample size. $E S$ was based on the change 
M.C. Arrieta-Leandro, J. Hernández-Elizondo, J. Jiménez-Díaz, High Intensity Interval Training in type 2 diabetes

between pre-test and post-test outcomes and calculated with the OpenMEE software [17] and the continuous random effects model [18]. Four studies did not report data necessary for meta-analysis and the authors were successfully contacted via e-mail to retrieve the information. Also, 4 articles reported HbA1c in $\mathrm{mmol} / \mathrm{l}$; to calculate $E S$, these values were converted to \% with a German DiaSys Diagnostic Systems GmbH converter [19].

\section{Quality assessment and risk of bias}

Quality was assessed with the PEDro scale [20]. The results were exposed to description purposes and evaluated as a moderator variable to look for possible trends depending on quality results. Heterogeneity analysis was performed with $\mathrm{I}^{2}$ in the OpenMEE software, subjective risk of bias was assessed with a funnel plot analysis in OpenMEE [17], and an objective analysis was conducted with Egger regression by using RStudio [21].

\section{Sensitivity analysis}

One study seemed to alter data heterogeneity, so analysis was performed with and without this trial to show the reader its impact on the results (see Figure 2b, c).
Moderator variable analysis

Moderator variables were assessed to find any possible factor affecting directly the $\mathrm{HbA1c}$ behaviour in the studies analysed. The categorical variables were meta-analysed with subgroup analysis and continuous variables were analysed with meta-regression, both in the OpenMEE software [17].

\section{Ethical approval}

The conducted research is not related to either human or animal use.

\section{Results}

A total of 233 studies were obtained from the different databases; 17 were added from other sources like reviews and research application updates. Among these 250 studies, 89 were duplicated records so a total of 161 articles were screened and compared with the inclusion criteria. After all the assessment, 22 studies were selected for meta-analysis (Figure 1).

A total of 564 participants were included in the analysis; among them, 361 performed the HIIT protocol. Gender analysis was not possible because most of the studies did not report the results distributed by gender. The main information about the included pa-
Records identified through database searching EBSCOHost: 24, Web of Science: 119 PubMed: 81, Scopus: 7, EMBASE: 2 Total: $n=233$

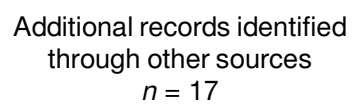

Figure 1. Flow diagram of the study selection process

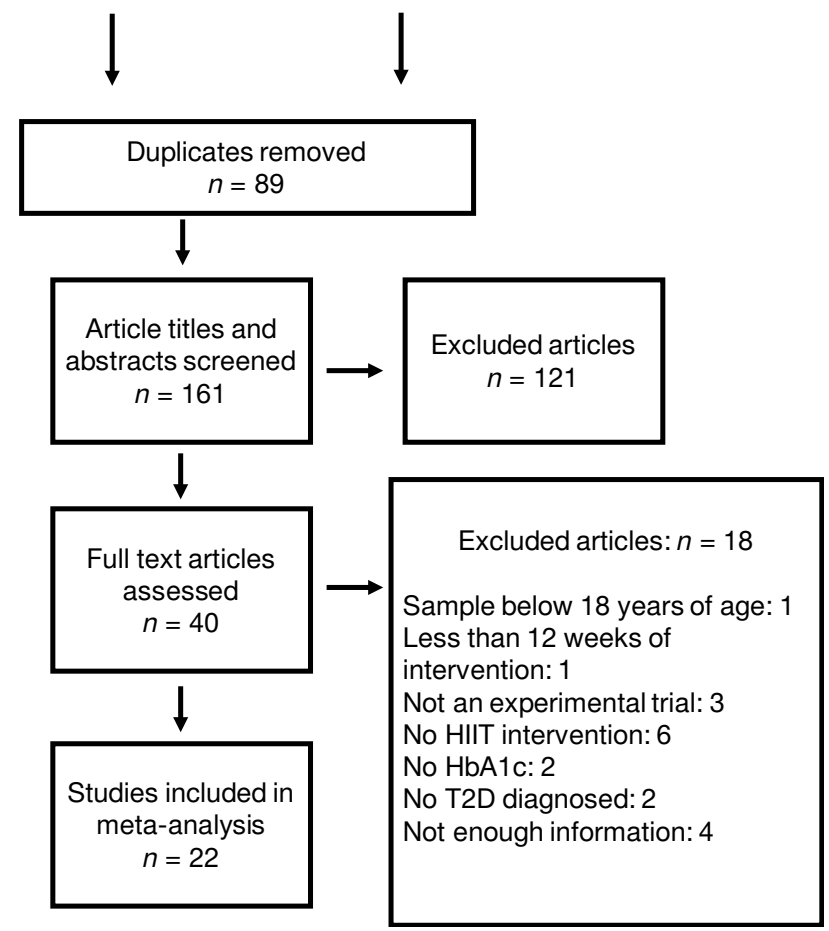


a)

\section{Studies}

Ahmed et al, 2019

Alvarez et al, 2016

Cassidy et al, 2014

Cassidy et al, 2016

Cassidy et al, 2018

Hua et al, 2019

Karstoft et al, 2013

Magalhaes et al, 2010

Mir et al, 2020

Mitranum et al, 2014

Subgroup Control (I^2=27.31\%, P=0.193)

Bellia et al, 2017

Magiamarchi et al, 2017

Way et al, 2020

Subgroup Control $+\left(\left.\right|^{\wedge} 2=54.9 \%, P=0.109\right)$

Overall (|^2=29.93\%, P=0.145)
Mean difference $95 \% \mathrm{CI}$

$0.554 \quad(-0.077,1.186)$ $0.212(-0.667,1.090)$ $0.069(-0.767,0.905)$ $0.316(-0.525,1.157)$ $0.906(0.029,1.784)$ $-0.114(-0.492,0.263)$ $1.483(0.377,2.590)$ $0.092(-0.499,0.683)$ $0.092(-0.499,0.683)$ $0.127(-0.589,0.843)$ $0.294(0.027,0.561$

$-0.529(-1.379,0.322)$ $0.000(-0.877,0.877)$ $0.770(-0.097,1.636)$ $0.077(-0.666,0.820)$ $0.259(0.011,0.508)$

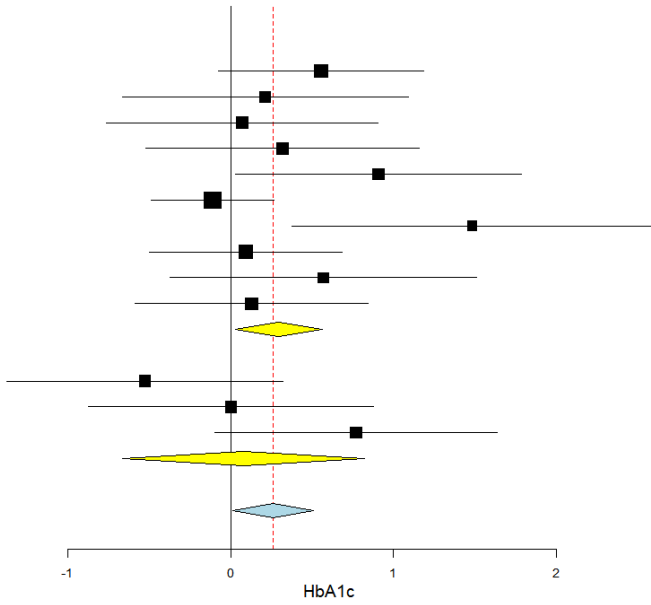

b)

\section{Studies}

Aguilera et al, 2015 Ahmed et al, 2019 Alvarez et al, 2016 Bellia et al, 2017 Cassidy et al, 2014 Cassidy et al, 2016 Cassidy et al, 2018 Francois et al, 2017 Hollekim-Strand et al, 2014 Hua et al, 2019 Karstoft et al, 2013 Macias Cervantes et al, 2017 Magiamarchi et al, 2017 Magiamarchi et al, 2017 Maillard et al, 2016 Mitranum et al, 2014 Stoa et al, 201 Way et al, 202 Subgroup HIIT $\left(I^{\wedge} 2=79.34 \%, P=0.000\right)$ Magalhaes et al, 2019 Mir et al, 2020 Sudarsono et al, 2019 Terada et al, 2013 Wormgoor et al, 2018 Subgroup HIIT + other $\left(I^{\wedge} 2=0 \%, P=0.843\right)$ Overall ( $\left.\left.\right|^{\wedge} 2=75.18 \%, P=0.000\right)$

\section{Mean difference $95 \%$ CI}

$-0.171(-1.153,0.811)$ $-1.426(-2.120,-0.732)$ $\begin{array}{rrr}-2.114 & (-3.073, & -1.154) \\ -0.816 & (-1.686, & 0.054)\end{array}$ $\begin{array}{lll}-0.816 & (-1.686, & 0.054) \\ -0.123 & (-0.923, & 0.678)\end{array}$ $\begin{array}{lll}-0.123(-0.923, & 0.678) \\ -0.304(-1.109, & 0.500)\end{array}$ $\begin{array}{lll}-0.304 & (-1.109, & 0.500)\end{array}$ $-0.248(-0.630,0.134)$ $-0.370(-0.995,0.255)$ $-2.431(-2.910,-1.951)$ $-0.379(-1.186, \quad 0.429)$ $-0.651(-1.472,0.170)$ $-0.897(-1.866,0.072)$ $-0.315(-1.301,0.671)$ $-0.211(-0.954,0.532)$ $-0.459(-1.161,0.243)$ $0.234(-1.037,0.569)$ $-0.713(-1.103,-0.322)$ $0.033(-0.735,0.802)$ $-0.569(-1.463,0.325)$ $-0.448(-1.109,0.214)$ $-0.169(-1.219,0.880)$

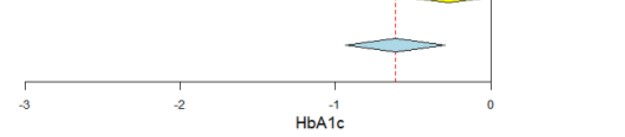

c)

Studies

Aguilera et al, 2015 Ahmed et al, 2019 Alvarez et al, 2016 Bellia et al, 2017 Cassidy et al, 2014 Cassidy et al, 2016 Cassidy et al, 2018 Francois et al, 2017 Hollekim-Strand et al, 2014 Karstoft et al, 2013 Macias Cervantes et al, 2017 Magiamarchi et al, 2017 Maillard et al, 2016 Mitranum et al, 2014 Stoa et al, 2017 Way et al, 2020

Subgroup HIIT (|^2=37.75\% , P=0.063)

Magalhaes et al, 2019 Mir et al, 2020

Sudarsono et al, 201

Terada et al, 2013

Wormgoor et al, 2018

Subgroup HIIT + other (I^ $2=0 \%, P=0.843)$

Overall (|^2=25.71\%, P=0.137)
Mean difference $95 \%$ CI

$-0.171(-1.153,0.811)$ $-1.426(-2.120,-0.732)$ $-2.114(-3.073,-1.154)$ $-0.816(-1.686,0.054)$ $-0.123(-0.923,0.678)$ $-0.304(-1.109,0.500)$ $-0.833(-1.704,0.038)$ $-0.835(-1.704,0.038)$ $0.370(-0.995,0.255)$ $-0.379(-1.186,0.429)$ $-0.651 \quad(-1.472,0.170)$ $-0.897(-1.866,0.072)$ $-0.315(-1.301,0.671)$ $-0.211(-0.954,0.532)$ $-0.459(-1.161,0.243)$ $-0.234(-1.037,0.569)$ $-0.560(-0.803,-0.317)$

$0.033(-0.735,0.802)$ $-0.569(-1.463,0.325)$ $-0.448(-1.109,0.214)$ $0.169(-1.219,0.880)$ $-0.163(-0.964,0.639)$

$-0.488(-0.684,-0.292)$

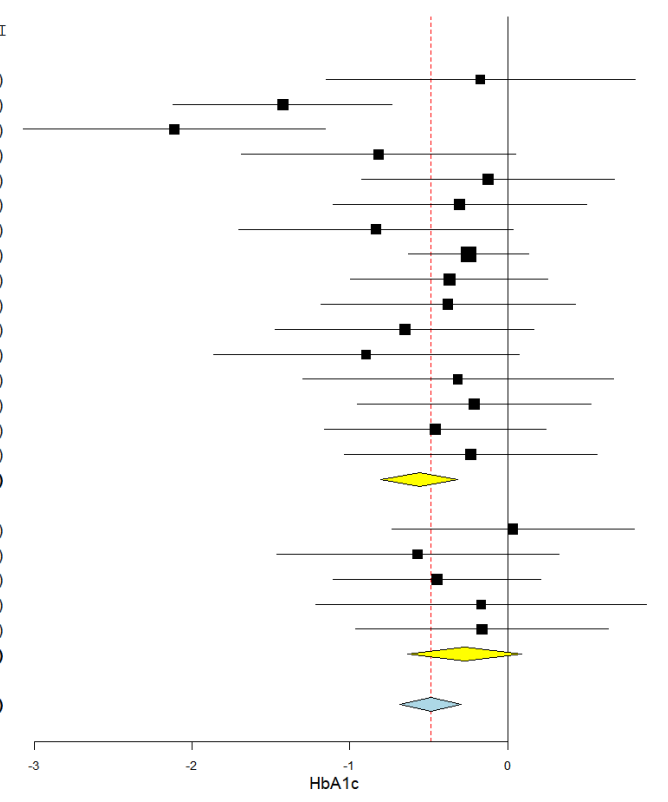

HbA1c - glycosylated haemoglobin, subgroup control - pure sedentary control group, subgroup control +control group with nutritional education or with low-level activity subgroup HIIT - group with High Intensity Interval Training subgroup HIIT + other - group with High Intensity Interval Training mixed with other type of training

Figure 2

(a) Forest plot for control groups and the effect on $\mathrm{HbA1c}$.

(b) Forest plot for experimental groups effect on HbA1c.

(c) Forest plot for experimental groups without the study by Hua et al. [31] 


\section{HUMAN MOVEMENT}

M.C. Arrieta-Leandro, J. Hernández-Elizondo, J. Jiménez-Díaz, High Intensity Interval Training in type 2 diabetes

Table 1. Main characteristics of the included papers

\begin{tabular}{|c|c|c|c|c|c|c|c|c|c|c|}
\hline \multirow[b]{2}{*}{ Article, year } & \multirow[b]{2}{*}{ Country } & \multicolumn{5}{|c|}{ Main characteristics of the subjects } & \multicolumn{4}{|c|}{ Intervention } \\
\hline & & $n$ & Sex & $\begin{array}{c}\text { Mean age } \\
\text { (years) }\end{array}$ & Medication & $\begin{array}{c}\text { Activity } \\
\text { level }\end{array}$ & $\begin{array}{l}\text { Duration of } \\
\text { intervention }\end{array}$ & $\begin{array}{l}\text { Frequency } \\
\text { of exercise }\end{array}$ & Method & Diet \\
\hline $\begin{array}{l}\text { Aguilera Eguía } \\
\text { et al., } 2015 \\
\text { [22] }\end{array}$ & Chile & 8 & Mixed & 62 & NR & NR & 12 weeks & $\begin{array}{l}5 \text { times/ } \\
\text { week }\end{array}$ & $\begin{array}{c}\text { Treadmill and cycle } \\
\text { ergometry }\end{array}$ & $\begin{array}{c}\text { Not } \\
\text { controlled }\end{array}$ \\
\hline \multirow{2}{*}{$\begin{array}{l}\text { Ahmed et al., } \\
2019 \text { [23] }\end{array}$} & \multirow{2}{*}{ Egypt } & $20^{*}$ & \multirow{2}{*}{ Male } & $51.8^{*}$ & \multirow{2}{*}{ Pill } & \multirow{2}{*}{ Sedentary } & \multirow{2}{*}{12 weeks } & \multirow{2}{*}{$\begin{array}{l}3 \text { times/ } \\
\text { week }\end{array}$} & \multirow{2}{*}{ Treadmill } & \multirow{2}{*}{$\begin{array}{c}\text { Not } \\
\text { controlled }\end{array}$} \\
\hline & & 20 & & 52.4 & & & & & & \\
\hline \multirow{2}{*}{$\begin{array}{l}\text { Alvarez et al., } \\
2016[24]\end{array}$} & \multirow{2}{*}{ Brazil } & $10^{*}$ & \multirow{2}{*}{ Mixed } & $43.1^{*}$ & \multirow{2}{*}{ Pill } & \multirow{2}{*}{ Sedentary } & \multirow{2}{*}{16 weeks } & \multirow{2}{*}{$\begin{array}{l}3 \text { times/ } \\
\text { week }\end{array}$} & \multirow{2}{*}{$\begin{array}{l}\text { Walking/jogging/ } \\
\text { running }\end{array}$} & \multirow{2}{*}{$\begin{array}{l}\text { Standardized } \\
\text { or controlled }\end{array}$} \\
\hline & & 13 & & 45.6 & & & & & & \\
\hline \multirow{2}{*}{$\begin{array}{l}\text { Bellia et al., } \\
2017 \text { [25] }\end{array}$} & \multirow{2}{*}{ Italy } & $11^{+}$ & \multirow{2}{*}{ - Mixed } & $56.3^{+}$ & \multirow{2}{*}{ Pill } & \multirow{2}{*}{ Sedentary } & \multirow{2}{*}{12 weeks } & \multirow{2}{*}{$\begin{array}{c}2.75 \text { times/ } \\
\text { week }\end{array}$} & $T$ & Standardized \\
\hline & & 11 & & 58.8 & & & & & 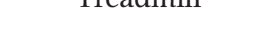 & or controlled \\
\hline Cassidy et al., & United & $11^{*}$ & Mivod & $60 *$ & ND & NDP & 12 strol & 3 times/ & 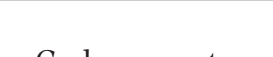 & $\mathrm{DP}$ \\
\hline $2014[26]$ & Kingdom & 12 & Mixed & 60 & NR & NR & 12 weeks & week & Cycle ergometry & NR \\
\hline Cassidy et al., & United & $14^{*}$ & - Mixed & $59 *$ & Pill & Sedentary & 12 weeks & 3 times/ & Cucle eroometry & Standardized \\
\hline 2016 [27] & Kingdom & 14 & vinxed & 61 & FIII & sedentary & 12 weeks & week & сусте ergometry & or controlled \\
\hline Cassidy et al., & United & $11^{*}$ & Mivod & $59 *$ & Medicine + & NR & 12 r & 3 times/ & $C_{\text {U }}$ & Standardized \\
\hline 2019 [28] & Kingdom & 11 & Nuxed & 60 & lifestyle & NR & 12 weeks & week & Сусте ergometry & or controlled \\
\hline $\begin{array}{l}\text { Francois et al., } \\
2017 \text { [29] }\end{array}$ & Canada & 53 & Mixed & 57.7 & NR & NR & 12 weeks & $\begin{array}{l}3 \text { times/ } \\
\text { week }\end{array}$ & $\begin{array}{l}\text { Treadmill or cycle } \\
\text { ergometry } \\
\text { or elliptical } \\
\text { and elastic bands }\end{array}$ & $\begin{array}{l}\text { Experimental } \\
\text { condition }\end{array}$ \\
\hline $\begin{array}{l}\text { Hollekim- } \\
\text { Strand et al., } \\
2014[30]\end{array}$ & Norway & 20 & Mixed & 58.6 & NR & NR & 12 weeks & $\begin{array}{c}3 \text { times/ } \\
\text { week }\end{array}$ & NR & NR \\
\hline Hua et al., & China & $54^{*}$ & Mixed & $43.7^{*}$ & Medicine + & $\mathrm{NR}$ & 12 weeks & 3 times/ & $C$ Yole orrometra & Experimental \\
\hline 2020 [31] & & 58 & & 44.3 & lifectyle & & & week & & condition \\
\hline Karstoft et al., & Denmark & $8^{*}$ & Mixed & $57.1^{*}$ & Medicine + & Sedentary & 16 woolro & 5 times/ & Walking/jogging/ & Standardized \\
\hline 2013 [13] & & 12 & & 57.5 & 1. & seuertiary & & week & . & or controlled \\
\hline $\begin{array}{l}\text { Macías- } \\
\text { Cervantes et } \\
\text { al., } 2017 \text { [32] }\end{array}$ & Mexico & 12 & NR & 45.8 & NR & Sedentary & 16 weeks & $\begin{array}{l}3 \text { times/ } \\
\text { week }\end{array}$ & Cycle ergometry & NR \\
\hline Magalhães et & & $22^{*}$ & & $59^{*}$ & ND & NI & & 3 times/ & & \\
\hline al., 2019 [33] & Portugal & 13 & Mined & 56.7 & NK & NK & 52 weeks & week & e ergometry & NK \\
\hline $\begin{array}{l}\text { Maillard et al., } \\
2016 \text { [35] }\end{array}$ & France & 8 & Female & 68.2 & $\begin{array}{l}\text { Pill and } \\
\text { injectable }\end{array}$ & Low & 16 weeks & $\begin{array}{l}2 \text { times/ } \\
\text { week }\end{array}$ & Cycle ergometry & $\begin{array}{l}\text { Standardized } \\
\text { or controlled }\end{array}$ \\
\hline Mangiamarchi & Chile & $10^{-}$ & Female & $54.1^{-}$ & Pill and & Sedentary & 12 weeks & 3 times/ & Cycle ergometry & Experimental \\
\hline [34] & & 9 & & 57.6 & in & & & & & 然 \\
\hline Mir et al., & Jran & $9^{*}$ & Male & $57.7^{*}$ & Pill & Sedentary & 12 & 3 times/ & $T r o$ & Not \\
\hline 2020 [36] & Iran & 10 & Mrale & 58.9 & Fill & sedentary & 12 weeks & & Ireaamint & controlled \\
\hline
\end{tabular}




\begin{tabular}{|c|c|c|c|c|c|c|c|c|c|c|}
\hline \multirow{2}{*}{$\begin{array}{l}\text { Mitranum } \\
\text { et al., } 2014 \\
\text { [37] }\end{array}$} & \multirow{2}{*}{ Thailand } & $15^{*}$ & \multirow{2}{*}{ Mixed } & $60.9^{*}$ & \multirow{2}{*}{ Pill } & \multirow{2}{*}{ Sedentary } & \multirow{2}{*}{12 weeks } & \multirow{2}{*}{$\begin{array}{c}3 \text { times/ } \\
\text { week }\end{array}$} & \multirow{2}{*}{ Treadmill } & \multirow{2}{*}{$\begin{array}{l}\text { Standardized } \\
\text { or controlled }\end{array}$} \\
\hline & & 14 & & 61.2 & & & & & & \\
\hline $\begin{array}{l}\text { Støa et al., } \\
2017 \text { [38] }\end{array}$ & Norway & 16 & NR & 59 & $\begin{array}{l}\text { Pill and } \\
\text { injectable }\end{array}$ & Sedentary & 12 weeks & $\begin{array}{l}3 \text { times/ } \\
\text { week }\end{array}$ & $\begin{array}{c}\text { Walking/jogging/ } \\
\text { running }\end{array}$ & $\begin{array}{l}\text { Standardized } \\
\text { or controlled }\end{array}$ \\
\hline $\begin{array}{l}\text { Sudarsono et } \\
\text { al., } 2019 \text { [39] }\end{array}$ & Indonesia & 18 & Mixed & 51.69 & Pill & NR & 12 weeks & $\begin{array}{l}3 \text { times/ } \\
\text { week }\end{array}$ & $\begin{array}{l}\text { Treadmill and } \\
\text { cycle ergometry }\end{array}$ & $\begin{array}{l}\text { Standardized } \\
\text { or controlled }\end{array}$ \\
\hline $\begin{array}{l}\text { Terada et al., } \\
2013 \text { [40] }\end{array}$ & Canada & 8 & Mixed & 62 & $\begin{array}{l}\text { Medicine + } \\
\text { lifestyle }\end{array}$ & Sedentary & 12 weeks & $\begin{array}{l}4 \text { times/ } \\
\text { week }\end{array}$ & $\begin{array}{l}\text { Treadmill and } \\
\text { cycle ergometry }\end{array}$ & $\begin{array}{l}\text { Standardized } \\
\text { or controlled }\end{array}$ \\
\hline \multirow{2}{*}{$\begin{array}{l}\text { Way et al., } \\
2020 \text { [41] }\end{array}$} & \multirow{2}{*}{ Australia } & $11^{+}$ & \multirow{2}{*}{ Mixed } & $51.9^{+}$ & \multirow{2}{*}{ Pill } & \multirow{2}{*}{ Sedentary } & \multirow{2}{*}{12 weeks } & \multirow{2}{*}{$\begin{array}{l}3 \text { times/ } \\
\text { week }\end{array}$} & \multirow{2}{*}{ Cycle ergometry } & \multirow{2}{*}{$\begin{array}{c}\text { Not } \\
\text { controlled }\end{array}$} \\
\hline & & 12 & & 56.9 & & & & & & \\
\hline $\begin{array}{l}\text { Wormgoor et } \\
\text { al., 2018 [42] }\end{array}$ & $\begin{array}{l}\text { New } \\
\text { Zealand }\end{array}$ & 12 & Male & 52.2 & $\begin{array}{l}\text { Pill and } \\
\text { injectable }\end{array}$ & NR & 12 weeks & $\begin{array}{l}3 \text { times/ } \\
\text { week }\end{array}$ & Cycle ergometry & $\begin{array}{l}\text { Standardized } \\
\text { or controlled }\end{array}$ \\
\hline
\end{tabular}

NR - not reported

${ }^{*}$ control group, ${ }^{+}$control + low activity level groups, ${ }^{-}$control + nutritional advice groups

pers can be found in Table 1. Figure 2 shows the subgroup analysis corresponding to control and experimental groups.

A total of 10 studies involved pure control sedentary groups, 3 trials had a control group mixed with another kind of treatment. For example, one of them included at least 10,000 steps/day or 70,000 steps/ week and another received nutritional education; because of this variety of the control groups, they were analysed in another subgroup. The results showed that being sedentary did not improve glycaemic control; on the contrary, HbA1c significantly increased in these cases, making glycaemic control worse. In the control + subgroup, glycaemic control did not present any changes (Figure 2a).

In the experimental group, ES was significantly lower. There were trials that involved only HIIT treatments and in 5 studies HIIT was mixed with another exercise protocol (e.g. combined with resistance training); these were analysed separately. The subgroup of HIIT revealed a significantly lower ES and the subgroup of HIIT + another intervention obtained lower $E S$ but the difference was not significant (Figure 2b). The 5 trials of HIIT + another category were not included in the moderator variable analysis.

The heterogeneity of the results was significantly high; a sensitivity analysis was performed and it showed that the study by Hua et al. [31] brought about important heterogeneity changes. When the forest plot was obtained without this study, $\mathrm{I}^{2}$ turned out non-significant (Figure 2c). Despite this, the results maintained significantly lower HbA1c when HIIT was performed.

The funnel plot subjective analysis shows that there is symmetry in data and the Egger regression test con-

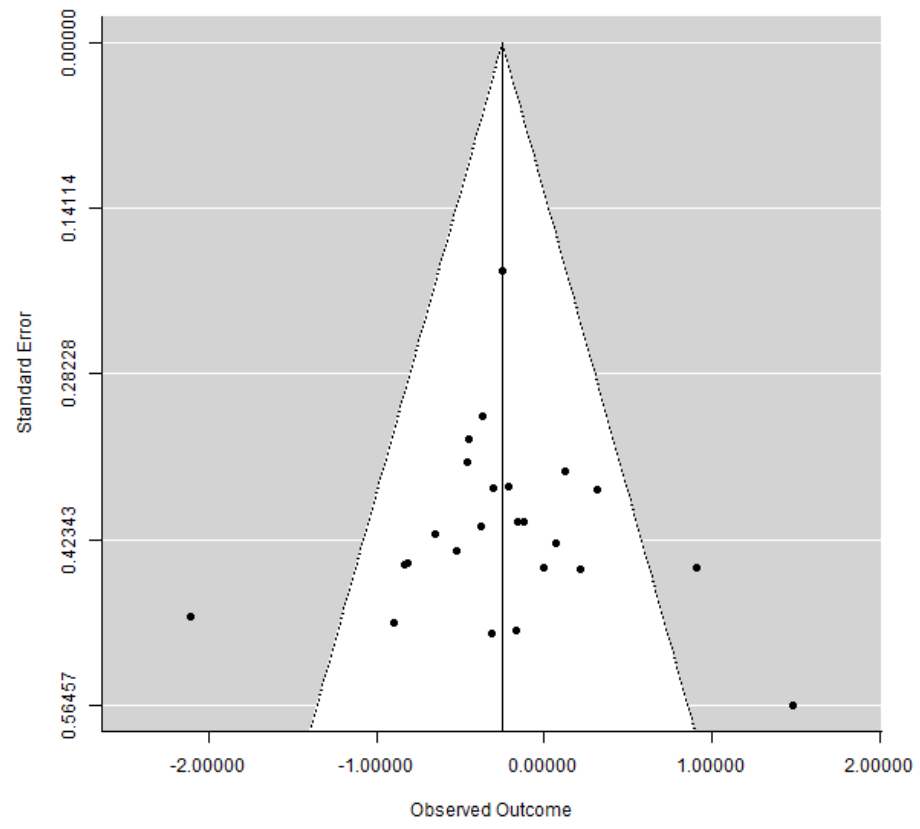

Figure 3. Funnel plot analysis of bias

firmed this in an objective way $(\mathrm{t}=-0.634, p=0.535$, slope: 0.179 ). This result indicates a low risk of bias in the studies included in the meta-analysis (Figure 3).

Quality assessment of the meta-analysed studies was performed with the PEDro scale [20] and is displayed in Table 2. There is an important detail about this evaluation. Because of the trials design, it was not possible to blind the subjects and therapists; for that reason, all the studies were assigned 0 in these items. This, however, does not necessarily mean that the risk of bias was increased. The meta-regression analysis revealed no significant trend in $E S$ depending on the quality score (Table 3); all 22 studies metaanalysed were included in this meta-regression. 


\section{HUMAN MOVEMENT}

M.C. Arrieta-Leandro, J. Hernández-Elizondo, J. Jiménez-Díaz, High Intensity Interval Training in type 2 diabetes

Table 2. Included trials quality assessment performed with PEDro scale

\begin{tabular}{|c|c|c|c|c|c|c|c|c|c|c|c|c|}
\hline Study & 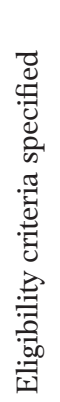 & 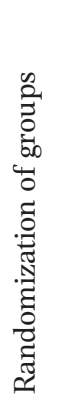 & 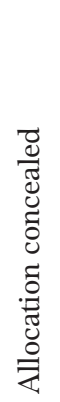 & 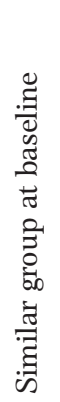 & 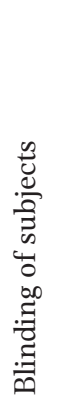 & 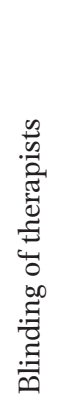 & 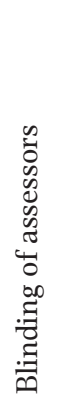 & 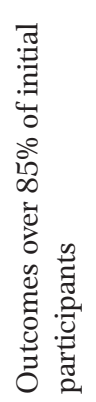 & 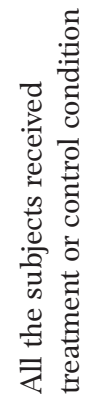 & 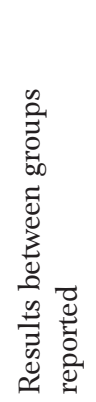 & 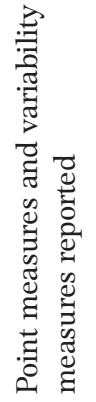 & Total \\
\hline Aguilera Eguía et al., 2015 [22] & 1 & 1 & 0 & ? & 0 & 0 & 1 & 1 & 0 & 1 & 1 & 6 \\
\hline Ahmed et al., 2019 [23] & 1 & 1 & 1 & $?$ & 0 & 0 & 0 & 0 & 1 & 1 & 1 & 6 \\
\hline Alvarez et al., 2016 [24] & 1 & 1 & 0 & 1 & 0 & 0 & 1 & 1 & 1 & 1 & 0 & 7 \\
\hline Bellia et al., 2017 [25] & 1 & 1 & 0 & 1 & 0 & 0 & 0 & 0 & 1 & 1 & 0 & 5 \\
\hline Cassidy et al., 2014 [26] & 1 & 1 & 0 & $?$ & 0 & 0 & $?$ & ? & 1 & 1 & 1 & 5 \\
\hline Cassidy et al., 2016 [27] & 1 & 1 & 1 & 1 & 0 & 0 & 0 & 0 & 1 & 1 & 1 & 7 \\
\hline Cassidy et al., 2019 [28] & 1 & 1 & 1 & 1 & 0 & 0 & 0 & 0 & 1 & 1 & 1 & 7 \\
\hline Francois et al., 2017 [29] & 1 & 1 & 0 & ? & 0 & 0 & 0 & 1 & 1 & 1 & 1 & 6 \\
\hline Hollekim-Strand et al., 2014 [30] & 1 & 1 & 0 & 1 & 0 & 0 & 0 & 0 & 1 & 1 & 1 & 6 \\
\hline Hua et al., 2020 [31] & 1 & 1 & 0 & 1 & 0 & 0 & 0 & 1 & 1 & 1 & 1 & 7 \\
\hline Karstoft et al., 2013 [13] & 1 & 1 & 0 & 1 & 0 & 0 & 1 & 1 & 1 & 1 & 1 & 8 \\
\hline Macías-Cervantes et al., 2017 [32] & 1 & 1 & 0 & 1 & 0 & 0 & 0 & ? & 1 & 1 & 1 & 6 \\
\hline Magalhães et al., 2019 [33] & 1 & 1 & 1 & 1 & 0 & 0 & 0 & 0 & 1 & 1 & 1 & 7 \\
\hline Maillard et al., 2016 [35] & 1 & 1 & 0 & 1 & 0 & 0 & 0 & 1 & 1 & 1 & 1 & 7 \\
\hline Mangiamarchi et al., 2017 [34] & 1 & 0 & 0 & 1 & 0 & 0 & 0 & 1 & 1 & 1 & 0 & 5 \\
\hline Mir et al., 2020 [36] & 1 & 1 & 0 & ? & 0 & 0 & 0 & 1 & 1 & 1 & 1 & 6 \\
\hline Mitranum et al., 2014 [37] & 1 & 1 & 0 & 1 & 0 & 0 & 0 & 1 & 1 & 1 & 1 & 7 \\
\hline Støa et al., 2017 [38] & 1 & 0 & 0 & 1 & 0 & 0 & 0 & 1 & 1 & 1 & 1 & 6 \\
\hline Sudarsono et al., 2019 [39] & 1 & 1 & 0 & 1 & 0 & 0 & 1 & 0 & 1 & 0 & 1 & 6 \\
\hline Terada et al., 2013 [40] & 1 & 1 & 1 & 1 & 0 & 0 & 0 & 1 & 1 & 1 & 1 & 8 \\
\hline Way et al., 2020 [41] & 1 & 1 & 1 & 1 & 0 & 0 & 0 & 1 & 1 & 1 & 1 & 8 \\
\hline Wormgoor et al., 2018 [42] & 1 & 1 & 1 & 1 & 0 & 0 & $?$ & 1 & 1 & 1 & 0 & 7 \\
\hline
\end{tabular}

0 - no, 1 - yes, ? - no clear information in the study

Table 3. Meta-regression results for continuous moderator variables

\begin{tabular}{lccr}
\hline Factor & $\beta$ & Data range & $p$ \\
\hline Age (years) & 0.096 & $44.3-68.2$ & $<0.01^{*}$ \\
Frequency of exercise & 0.162 & $2.75-5$ & 0.541 \\
$\quad$ (times/week) & & & \\
Number of intervals & 0.002 & $2.75-60$ & 0.912 \\
High interval duration (s) & 0.001 & $8-240$ & 0.528 \\
Low interval duration (s) & 0.004 & $12-240$ & 0.222 \\
Quality of studies & -0.048 & $5-8$ & 0.780 \\
$\quad$ (PEDro scale) & & & \\
$* p<0.05$ & & &
\end{tabular}

\section{Type of diet}

As for the diet intervention (Figure 4a), the studies with a standardized or controlled diet and those that did not control the diet presented significantly lower $E S$ than the studies that made diet part of the ex- perimental condition. It is important to highlight that there are few studies in the experimental condition category and this ES could not be accurate.

\section{Type of medication}

In the type of medication analysis (Figure 4b), all groups significantly improved HbA1c, regardless of the type of pharmacological treatment received by the participants. In the category where the studies included participants without pharmacological treatment, treated with lifestyle changes, no significant improvement was observed.

\section{Method of exercise}

In the subgroup analysis of exercise method, the cycle ergometer and the treadmill presented significantly lower ES (Figure 5a). 
a)

Studies

Aguilera et al, 2015

Ahmed et al, 2019

Alvarez et al, 2016

Way et al, 2020

Bellia et al, 2017

Cassidy et al, 2018

Karstoft et al, 2013

Maillard et al, 2016

Mitranum et al, 2014

Stoa et al, 2017

Subgroup Standardized or controlled $\left(I^{\wedge} 2=0 \%, P=0.866\right)$

Francois et al, 2017

Hua et al, 2019

Subgroup Experimental condition $\left(I^{\wedge} 2=95.9 \%, P=0.000\right)$

Overall $\left(I^{\wedge} 2=82.15 \%, P=0.000\right)$
Cassidy et al, 2016

Subgroup Not controlled $\left(I^{\wedge} 2=73.63 \%, P=0.004\right)$

Magiamarchi et al, 2017

Mean difference 95\% CI

$-0.171(-1.153,0.811)$

$-1.426(-2.120,-0.732)$

$-2.114(-3.073,-1.154)$

$-0.304(-1.109,0.500)$

$-0.234(-1.037,0.569)$

$-0.848(-1.578,-0.118)$

$-0.816(-1.686,0.054)$

$-0.833(-1.704,0.038)$

$-0.379(-1.186,0.429)$

$-0.315(-1.301,0.671)$

$-0.211(-0.954,0.532)$

$-0.459(-1.161,0.243)$

$-0.486(-0.819,-0.153)$

$-0.248 \quad(-0.630,0.134)$

$-2.431(-2.910,-1.951)$

$-0.897(-1.866,0.072)$

$-1.197(-2.757,0.363)$

$-0.784(-1.246,-0.322)$

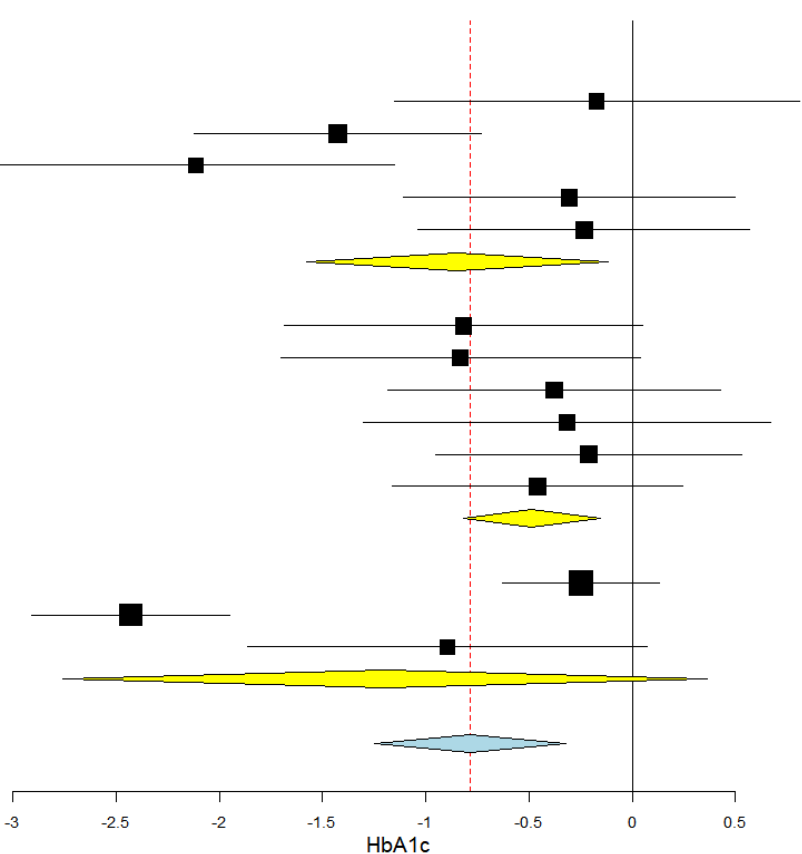

Mean difference 95\% CI

$-1.426(-2.120,-0.732)$

$-2.114(-3.073,-1.154)$

$-0.816(-1.686,0.054)$

$-0.304(-1.109,0.500)$

$-0.211(-0.954,0.532)$

$-0.234 \quad(-1.037,0.569)$

$-0.830(-1.420,-0.240)$

$-0.833(-1.704,0.038)$

$-2.431(-2.910,-1.951)$

$-0.379(-1.186,0.429)$

Hua et al, 2019

Karstoft et al, 2013

$-1.247(-2.635,0.141)$

Magiamarchi et al, 2017

Maillard et al, 2016

$-0.897(-1.866,0.072)$

$-0.315(-1.301,0.671)$

$-0.459(-1.161,0.243)$

$-0.536(-1.029,-0.044)$

$-0.883(-1.398,-0.368)$

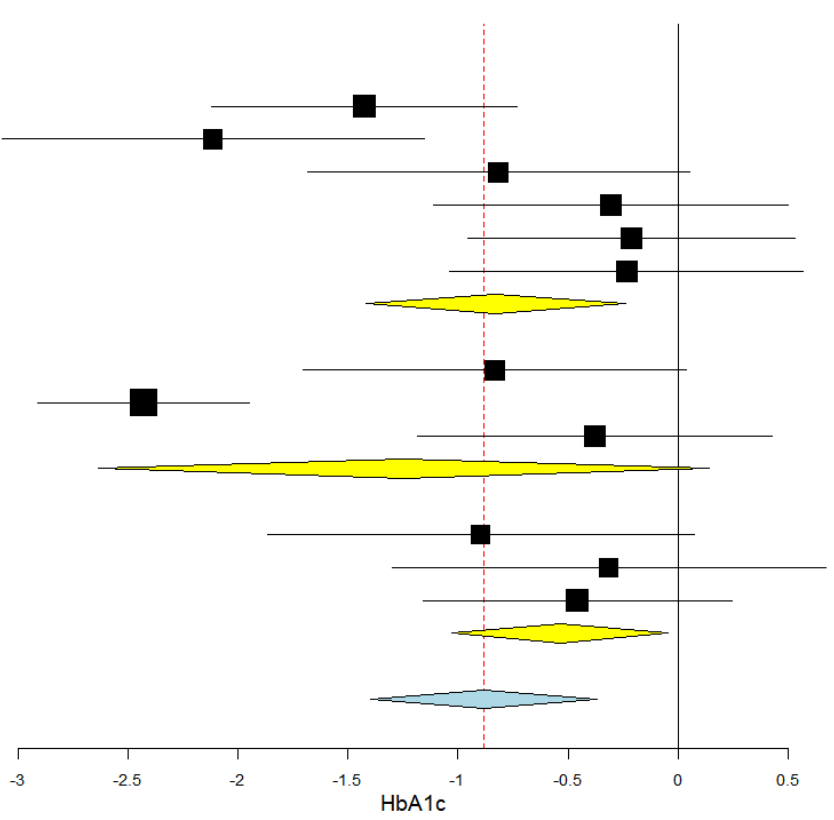

HbA1c - glycosylated haemoglobin

Figure 4. (a) Effect of High Intensity Interval Training on HbA1c depending on diet intervention.

(b) Effect of High Intensity Interval Training on HbA1c depending on received medication 


\section{HUMAN MOVEMENT}

M.C. Arrieta-Leandro, J. Hernández-Elizondo, J. Jiménez-Díaz, High Intensity Interval Training in type 2 diabetes

a) Studies

Aguilera et al, 2015

Subgroup treadmill and cycle ergometry $\left(I^{\wedge} 2=N A, P=N A\right)$

Ahmed et al, 2019

Bellia et al, 2017

Mitranum et al, 2014

Subgroup treadmill $\left(\left.\right|^{\wedge} 2=63.61 \%, P=0.064\right)$

Alvarez et al, 2016

Karstoft et al, 2013

Stoa et al, 2017

Subgroup walking/jogging/running $\left(\left(^{\wedge} 2=78.13 \%, P=0.010\right)\right.$

Cassidy et al, 2014

Cassidy et al, 2016

Cassidy et al, 2018

Hua et al, 2019

Macias Cervantes et al, 2017

Magiamarchi et al, 2017

Maillard et al, 2016

Way et al, 2020

Subgroup cycle ergometry $\left(\left.\right|^{\wedge} 2=85.28 \%, P=0.000\right)$

Francois et al, 2017

Subgroup treadmill or cycle ergometry or elliptical and elastic bands (I^2=NA , P=NA)

Overall ( $\left.\left.\right|^{\wedge} 2=80.22 \%, P=0.000\right)$
Mean difference $95 \% \mathrm{CI}$

$-0.171(-1.153,0.811)$ $-0.171(-1.153,0.811)$

$-1.426(-2.120,-0.732)$ $-0.816(-1.686,0.054)$ $-0.211(-0.954,0.532)$ $-0.828(-1.561,-0.095)$

$-2.114(-3.073,-1.154)$ $-0.379(-1.186, \quad 0.429)$ $-0.459(-1.161,0.243)$ $-0.943(-1.953,0.067)$

$-0.123(-0.923,0.678)$ $-0.304(-1.109,0.500)$ $-0.833(-1.704,0.038)$ $-2.431(-2.910,-1.951)$ $-0.651(-1.472,0.170)$ $-0.897(-1.866,0.072)$ $-0.315(-1.301,0.671)$ $-0.234(-1.037,0.569)$ $-0.749(-1.472,-0.026)$ $-0.248(-0.630,0.134)$ $-0.248(-0.630,0.134)$

$-0.736(-1.151,-0.320)$

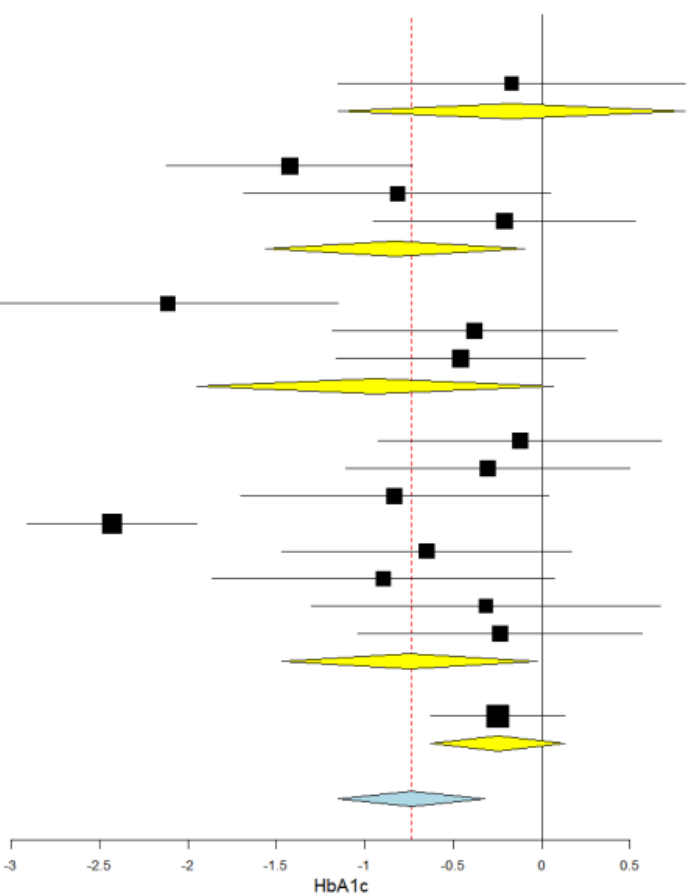

b)

Studies

Aguilera et al, 2015

Ahmed et al, 2019

Bellia et al, 2017

Cassidy et al, 2014

Cassidy et al, 2016

Cassidy et al, 2018

Francois et al, 2017

Hollekim-Strand et al, 2014

Hua et al, 2019

Magiamarchi et al, 2017

Mitranum et al, 2014

Stoa et al, 2017

Way et al, 2020

Subgroup 12 weeks (I^2=82.38\%, P=0.000)

Alvarez et al, 2016

Karstoft et al, 2013

Macias Cervantes et al, 2017

Maillard et al, 2016

Subgroup 16 weeks $\left(I^{\wedge} 2=67.73 \%, P=0.026\right)$

Overall (|^2=79.34 \%, P=0.000)

$-0.713(-1.103,-0.322)$

$-0.171(-1.153,0.811)$

$-1.426(-2.120,-0.732)$

$-0.816(-1.686,0.054)$

$-0.123(-0.923,0.678)$

$-0.304(-1.109,0.500)$

$-0.833(-1.704,0.038)$

$-0.248(-0.630,0.134)$

$-0.370(-0.995,0.255)$

$-2.431(-2.910,-1.951)$

$-0.897(-1.866,0.072)$

$-0.211(-0.954,0.532)$

$-0.459(-1.161,0.243)$

$-0.234(-1.037,0.569)$

$-0.672(-1.132,-0.213)$

$-2.114(-3.073,-1.154)$

$-0.379(-1.186,0.429)$

$-0.651(-1.472,0.170)$

$-0.315(-1.301,0.671)$

$-0.848(-1.631,-0.066)$

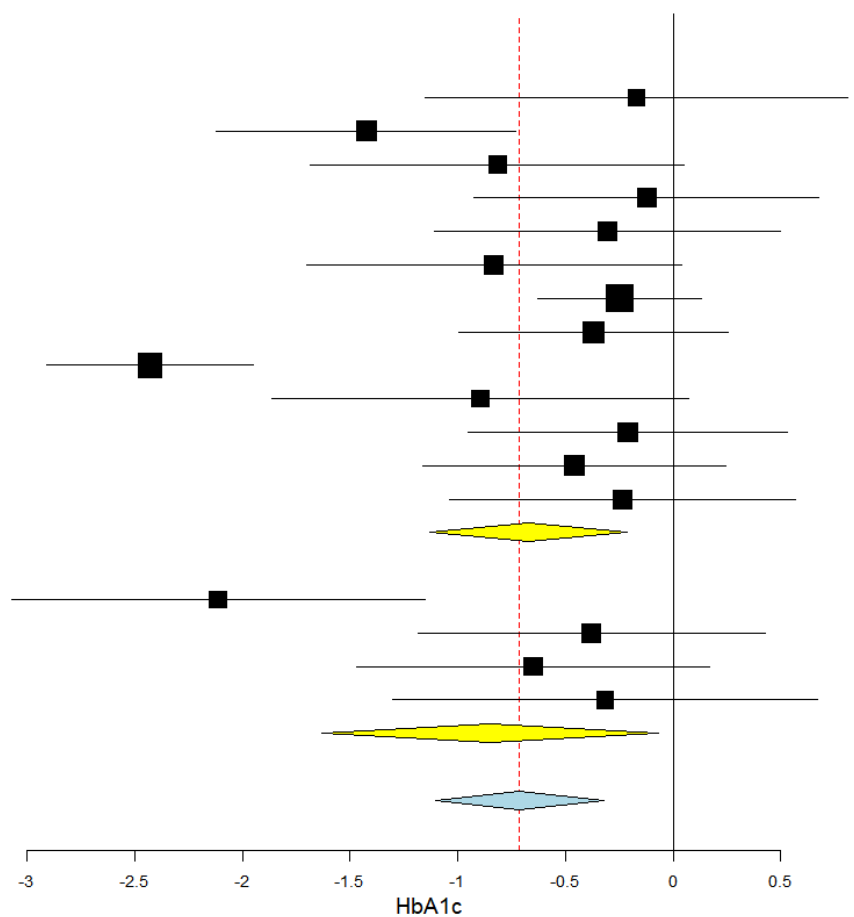

HbA1c - glycosylated haemoglobin

Figure 5. (a) Effect of High Intensity Interval Training on HbA1c depending on the type of exercise performed.

(b) Effect of High Intensity Interval Training on HbA1c depending on the number of intervention weeks 


\section{Intervention duration}

In the studies included, the interventions lasted 12 or 16 weeks; their duration was analysed as a categorical variable (Figure 5b). Trials that endured 16 weeks revealed a better improvement in $E S$ but both categories, as well as the overall group, presented significantly lower outcomes.

\section{High interval intensity}

The intensity of the high interval was measured with different physiological parameters in the analysed trials. For that reason, all the information concerning this topic was classified in accordance with the ACSM guidelines [43] as very light, light, moderate, vigorous, or maximal. Those studies that involved an intensity progress during the trial, the different intensities were averaged for analysis purposes. All the high intervals in the trials presented vigorous or maximal intensity (Figure 6), and ES was significantly lower in the overall analysis. In the subgroup analysis, the vigorous intensity kept significantly lower but the maximal subgroup was not significantly different.

The meta-regression analysis showed a significant impact of age on the results (Table 3). Regression presented a positive correlation: the older the participant, the smaller effect HIIT had on HbA1c $(E S=$ +0.096 for each subsequent year of age). The performance variables that revealed progression during the trial were averaged for analysis purposes. All other continuous variables showed no significant trends and their ranges are depicted in Table 3.

\section{Discussion}

The experimental HIIT group revealed a significant reduction in $E S$ of $\mathrm{HbA1c}$; in the trials that implemented only a HIIT protocol without any other training, $E S$ was bigger. Among these studies, those by Ahmed et al. [23], Alvarez et al. [24], and Hua et al. [31] presented the biggest changes $(E S=-1.426, E S=$ $-2.114, E S=-2.431$, respectively). Seven of the 17 only-HIIT studies had ES below the overall, but all the 17 studies indicated a negative size effect. This is consistent with the meta-analysis by Jang et al. [14], who found significant $E S$ when comparing the HIIT and control groups. On the contrary, Liu et al. [15] did not observe significant differences in HbA1c in comparison with controls, but noted a difference in comparison with continuous aerobic exercise; on the other hand, De Nardi et al. [11] did not find significant differences in comparison with continuous aerobic exercise. An important detail about these previous metaanalyses is that they involved fewer studies than this meta-analysis, and De Nardi et al. [11] included participants diagnosed with prediabetes; these can be possible reasons for the differences in the results.

\begin{tabular}{|c|c|c|}
\hline Studies & Mean difference & e $95 \% \mathrm{CI}$ \\
\hline Aguilera et al, 2015 & $-0.171(-1.153$ & $0.811)$ \\
\hline Alvarez et al, 2016 & $-2.114(-3.073$ & $-1.154)$ \\
\hline Subgroup Maximal $\left(I^{\wedge} 2=87 \%, P=0.006\right)$ & $-1.145(-3.049$ & $0.758)$ \\
\hline Ahmed et al, 2019 & $-1.426(-2.120$ & $-0.732)$ \\
\hline Bellia et al, 2017 & $-0.816(-1.686$ & $0.054)$ \\
\hline Cassidy et al, 2014 & $-0.123(-0.923$ & $0.678)$ \\
\hline Cassidy et al, 2016 & $-0.304 \quad(-1.109$ & $0.500)$ \\
\hline Cassidy et al, 2018 & $-0.833(-1.704$ & $0.038)$ \\
\hline Francois et al, 2017 & $-0.248 \quad(-0.630$ & $0.134)$ \\
\hline Hollekim-Strand et al, 2014 & $-0.370 \quad(-0.995$ & $0.255)$ \\
\hline Hua et al, 2019 & $-2.431(-2.910$ & $-1.951)$ \\
\hline Macias Cervantes et al, 2017 & $-0.651(-1.472$ & $0.170)$ \\
\hline Magiamarchi et al, 2017 & $-0.897(-1.866$ & $0.072)$ \\
\hline Maillard et al, 2016 & $-0.315(-1.301$ & $0.671)$ \\
\hline Stoa et al, 2017 & $-0.459 \quad(-1.161$ & $0.243)$ \\
\hline Subgroup Vigorous $\left(I^{\wedge} 2=82.67 \%, P=0.000\right)$ & $-0.755(-1.237$ & $-0.274)$ \\
\hline Overall $\left(I^{\wedge} 2=81.97 \%, P=0.000\right)$ & $-0.806(-1.260$ & $-0.351)$ \\
\hline
\end{tabular}

HbA1c - glycosylated haemoglobin

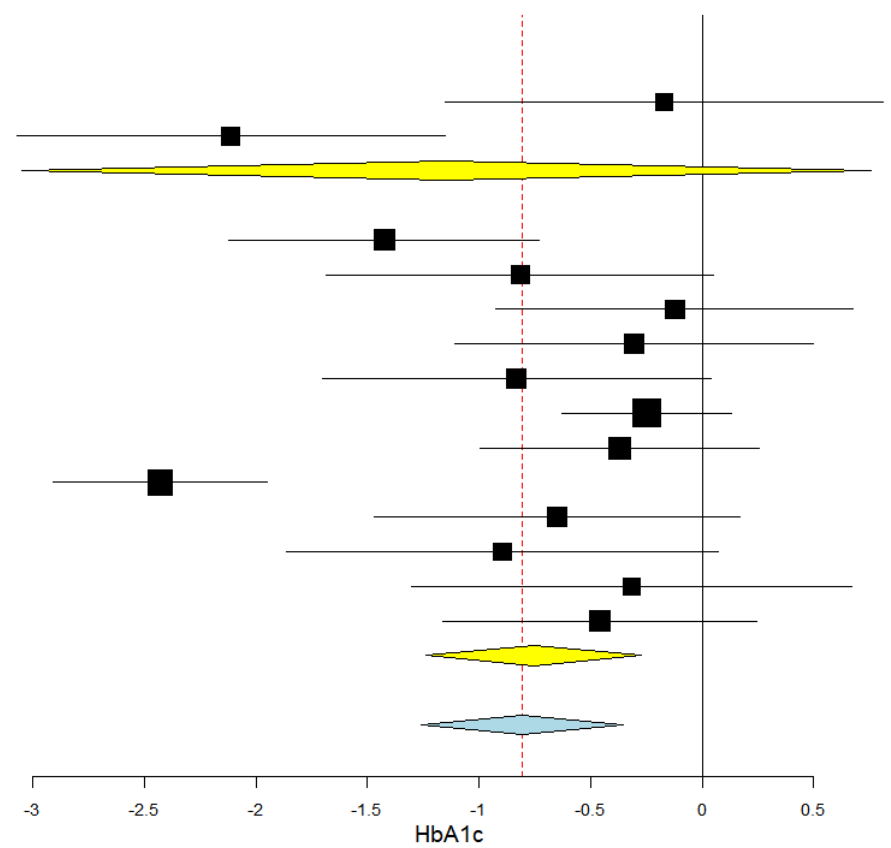

Figure 6. Effect of High Intensity Interval Training on HbA1c depending on the high interval intensity [43] 
M.C. Arrieta-Leandro, J. Hernández-Elizondo, J. Jiménez-Díaz, High Intensity Interval Training in type 2 diabetes

In some of the trials analysed, HIIT was complemented with another type of training and ES reduction was not significant, but there were only 5 studies applying this method. It is important to highlight that all of these 5 studies presented smaller ES than the overall and actually Magalhães et al. [33] implied positive $E S$ without significant changes. It is therefore necessary to perform more research to obtain a clear picture of the effect of HIIT complemented with some other training alternatives.

A recent meta-analysis showed a comparison between HIIT, moderate intensity training, low intensity training, and control in affecting HbA1c in T2D participants [44]. A significant reduction of HbA1c was found when HIIT was performed, as in the present meta-analysis. HIIT turned out more beneficial than control and low intensity training. There was no significant difference between HIIT and moderate intensity training [44].

A possible explanation for the $\mathrm{HbA} 1 \mathrm{c}$ reduction could be the effect of HIIT on appetite [45]; it was proved that HIIT reduced ghrelin plasma levels in obese diabetic subjects and also increased glucagonlike peptide- 1 concentrations in obese and normal weight diabetics. Ghrelin plasma levels reduction helps to improve appetite control and regulate energy intake. The improvement of glucagon-like peptide- 1 concentrations enhances insulin metabolism. Both factors can contribute to $\mathrm{HbA1c}$ reduction [46, 47].

A significant increase of $E S$ was observed in the control groups, corresponding with a negative effect of sedentarism on diabetes control. The study by Karstoft et al. [13] presented the biggest $E S(+1.483)$, and only Hua et al. [31] indicated negative ES (-0.114) between the sedentary control group studies. There were 3 trials that had control groups mixed with nutritional education or low-level activity; these did not reveal significant changes, whereas the pure sedentary control group had a significant increase in ES. The results show that being sedentary not only is unsuitable to improve glycaemic control, but also worsens the status of this chronic disease. This confirms the importance of an integrated treatment (diet, medication, and exercise) to obtain better glycaemic control, already reported in the literature [14].

With reference to the moderator variable analysis, there are some aspects to highlight. Concerning the diet, when it was controlled or standardized or the participants were instructed to continue their usual habits without any control, the results were better than when diet constituted part of the experimental condition. This reflects the influence of diet on T2D treat- ment. In the case of the type of medication, all the pharmacologic alternatives presented a significant ES reduction but when participants with lifestyle modification without medication were included, the results were not satisfactory. These facts again imply the importance of an integrated treatment where it is necessary to combine healthy lifestyle with exercise and pharmacologic treatment for better T2D control [48].

Sex analysis was not possible because of lack of appropriate information in the studies meta-analysed. A study published as an ACSM meeting abstract found a bigger reduction of $\mathrm{HbA1c}$ in males when they followed an aerobic training, resistance training, and flexibility training program [49].

The other moderator variable analysis showed that the best HIIT program involved a cycle ergometer or treadmill used for at least 12 weeks; better results were achieved with 16 weeks and with the high interval in vigorous intensity in accordance with ACSM guidelines [43]. Maximal intensity did not imply significant results; as it only appeared in 2 studies, further research is needed to draw conclusions about maximal intensity.

The meta-regression analysis showed no trend concerning exercise frequency, number of intervals, or interval duration; so, when the protocol was prescribed in the ranges displayed in Table 3, the result in $\mathrm{HbA1c}$ was satisfactory. The analysis revealed a significant trend with reference to age: the older the person, the smaller reduction in $\mathrm{HbA1c}$ the $E S$ indicated. In the study by Hua et al. [31], the average age was 44.3 years, and, the lowest in this meta-analysis, its $E S$ equalled -2.431 . On the other hand, Maillard et al. [35] involved the oldest participants, with an average age of 68.2 years, and $E S$ was -0.315 . It illustrates the trend found in the meta-regression results.

A recent review indicated that HIIT could be beneficial for glycaemic control in patients of any age; however, it remains unclear if this benefit is the same for all ages, so further investigation is recommended to make a specific evaluation concerning the participants' age [50]. That prediction is consistent with this metaregression analysis, which found significant differences in HbA1c reduction depending on age.

Among the strengths of this meta-analysis, there is the quality analysis, performed in accordance with the PEDro scale (with the assumption that it was not possible to blind the subjects and therapists because of the design of the studies). There is a low risk of bias in the trials analysed, so the results are reliable. Moreover, as revealed in the meta-regression analysis (Table 3), there is no influence of the quality score on the 
results. Another strength of this study is that papers published in Spanish or English were included and there was no publishing year restriction in the search.

On the other hand, this meta-analysis included T2D-diagnosed participants only; prediabetes individuals were excluded. The intervention length was filtered in accordance with the newest recommendations for HbA1c assessment (at least 12 weeks) [7, 8]. In addition, to our knowledge, this is the only metaanalysis evaluating moderator variables and proposing a HIIT protocol.

Among the limitations of this study, there are design weaknesses, lack of control groups in many trials, and lack of randomization in 2 studies. In the moderator variable analysis, it was not possible to include all the information because there was not enough information in some studies. Furthermore, some trial designs combined HIIT and other training modalities; these could not be included in the moderator variable analysis. There was another case with control groups in which nutritional education, diet, or another exercise were part of the intervention; this made them nonstrict control groups.

\section{Conclusions}

HIIT has a beneficial effect on HbA1c in people diagnosed with T2D and this effect is influenced by diet, training program intensity, and age. Keeping sedentary despite medical treatment can worsen glycaemic control. It is important to take into account the frequency, intensity, and length of intervention, interval duration, and the number of intervals when making prescription; this should be done by qualified professionals.

HIIT can be applied in people with T2D and medical approval; the suggested regimen involves practising 2-5 times/week, with vigorous intensity intervals lasting from 8 seconds to 4 minutes, for at least 12 weeks, on a treadmill or cycle ergometer.

\section{Acknowledgements}

We would like to thank all the professors and master program participants who advised us on improving this meta-analysis.

\section{Disclosure statement}

No author has any financial interest or received any financial benefit from this research.

\section{Conflict of interest}

The authors state no conflict of interest.

\section{References}

* Studies meta-analysed

1. Franz MJ, Evert AB. Medical nutrition therapy for diabetes mellitus and hypoglycemia of nondiabetic origin. In: Mahan LK, Raymond JL (eds.), Krause's food \& the nutrition care process. St. Louis: Elsevier; 2017; 586-618.

2. Zatońska K, Basiak-Rasała A, Rózańska D, Karczewski M, Wołyniec M, Szuba A, et al. Changes in diabetes prevalence and corresponding risk factors - findings from 3- and 6-year follow-up of PURE Poland cohort study. BMC Public Health. 2020;20(1):843; doi: 10.1186/s12889-020-08970-5.

3. Aguilar AY. Costa Rican people lose an average of 13.2 years of life because of diabetes [in Spanish]. 2018. Available from: https://amprensa.com/2018/09/costarricenses-pierde-en-promedio-13-2-anos-de-vidaa-causa-de-la-diabetes/.

4. Buresh R, Berg K. Exercise for the management of type 2 diabetes mellitus: factors to consider with current guidelines. J Sports Med Phys Fitness. 2018;58(4):510524; doi: 10.23736/S0022-4707.17.06969-9.

5. World Health Organization. Use of glycated haemoglobin (HbA1c) in the diagnosis of diabetes mellitus. Geneva: WHO; 2011. Available from: https://www.who. int/diabetes/publications/report-hba1c_2011.pdf.

6. Shrivastava A, Mishra P, Shrivastava N, Mishra S, Kumar V, Zaidi SNH, et al. Awareness of glycosylated haemoglobin (HbA1c) among type 2 diabetes mellitus patients in a diabetic clinic in urban Allahabad. HECS Int J Comm Health Med Res. 2018;4(2):61-64.

7. Morgen EK, Naugler C. Inappropriate repeats of six common tests in a Canadian city: a population cohort study within a laboratory informatics framework. Am J Clin Pathol. 2015;144(5):704-712; doi: 10.1309/ AJCPYXDAUS2F8XJY.

8. Reynolds TM, Smellie WSA, Twomey PJ. Glycated haemoglobin (HbA1c) monitoring. BMJ. 2006;333(7568): 586-588; doi: 10.1136/bmj.38947.627847.AE.

9. Da Silva DE, Grande AJ, Roever L, Tse G, Liu T, Biondi-Zoccai G, et al. High-intensity interval training in patients with type 2 diabetes mellitus: a systematic review. Curr Atheroscler Rep. 2019;21(2):8; doi: 10.1007/ s11883-019-0767-9.

10. Wormgoor SG, Dalleck LC, Zinn C, Harris NK. Effects of high-intensity interval training on people living with type 2 diabetes: a narrative review. Can J Diabetes. 2017;41(5):536-547; doi: 10.1016/j.jcjd.2016.12.004.

11. De Nardi AT, Tolves T, Lenzi TL, Signori LU, da Silva AMV. High-intensity interval training versus continuous training on physiological and metabolic variables in prediabetes and type 2 diabetes: a meta-analysis. Diabetes Res Clin Pract. 2018;137:149-159; doi: 10.1016/ j.diabres.2017.12.017.

12. Deguchi K, Enishi T, Sato N, Miura H, Fujinaka Y, Matsuhisa M, et al. Acute effect of fast walking on postprandial blood glucose control in type 2 diabetes. 
M.C. Arrieta-Leandro, J. Hernández-Elizondo, J. Jiménez-Díaz, High Intensity Interval Training in type 2 diabetes

Diabetol Int. 2016;7(2):119-123; doi: 10.1007/s13340015-0217-z.

13. *Karstoft K, Winding K, Knudsen SH, Nielsen JS, Thomsen C, Pedersen BK, et al. The effects of free-living interval-walking training on glycemic control, body composition, and physical fitness in type 2 diabetic patients: a randomized, controlled trial. Diabetes Care. 2013;36(2):228-236; doi: 10.2337/dc12-0658.

14. Jang JE, Cho Y, Lee BW, Shin ES, Lee SH. Effectiveness of exercise intervention in reducing body weight and glycosylated hemoglobin levels in patients with type 2 diabetes mellitus in Korea: a systematic review and meta-analysis. Diabetes Metab J. 2019;43(3):302318; doi: 10.4093/dmj.2018.0062.

15. Liu J-X, Zhu L, Li P-J, Li N, Xu Y-B. Effectiveness of high-intensity interval training on glycemic control and cardiorespiratory fitness in patients with type 2 diabetes: a systematic review and meta-analysis. Aging Clin Exp Res. 2019;31(5):575-593; doi: 10.1007/ s40520-018-1012-z.

16. Liberati A, Altman DG, Tetzlaff J, Mulrow C, Gøtzsche PC, Ioannidis JPA, et al. The PRISMA statement for reporting systematic reviews and meta-analyses of studies that evaluate healthcare interventions: explanation and elaboration. BMJ. 2009;339:b2700; doi: 10.1136/ bmj.b2700.

17. Wallace BC, Lajeunesse MJ, Dietz G, Dahabreh IJ, Trikalinos TA, Schmid CH, et al. OpenMEE: intuitive, open-source software for meta-analysis in ecology and evolutionary biology. Methods Ecol Evol. 2017;8(8): 941-947; doi: 10.1111/2041-210X.12708.

18. Borenstein M, Higgins JPT, Hedges LV, Rothstein HR. Basics of meta-analysis: $\mathrm{I}^{2}$ is not an absolute measure of heterogeneity. Res Synth Methods. 2017;8(1):5-18; doi: 10.1002/jrsm.1230.

19. DiaSys Diagnostic Systems. HbA1c calculator. Available from: https://www.hba1cnet.com/hba1c-calculator/.

20. Physiotherapy Evidence Database. PEDro scale. Available from: https://www.pedro.org.au/english/downloads/pedro-scale/.

21. RStudio Team. RStudio: integrated development for R. Boston: RStudio, Inc.; 2015. Available from: http:// www.rstudio.com/.

22. *Aguilera Eguía RA, Russell Guzmán JA, Soto Muñoz ME, Villegas González BE, Poblete Aro CE, Ibacache Palma A. Effect of high-intensity interval training on the reduction of glycosylated hemoglobin in type-2 diabetic adult patients [in Spanish]. Medwave. 2015;15(2): e6079; doi: 10.5867/medwave.2015.02.6079.

23. *Ahmed AS, Ahmed MS, Mahmoud WS, Abdelbasset WK, Elnaggar RK. Effect of high intensity interval training on heart rate variability and aerobic capacity in obese adults with type 2 diabetes mellitus. Biosci Res. 2019;16(3):2450-2458.

24. *Alvarez C, Ramirez-Campillo R, Martinez-Salazar C, Mancilla R, Flores-Opazo M, Cano-Montoya J, et al. Low-volume high-intensity interval training as a ther- apy for type 2 diabetes. Int J Sports Med. 2016;37(9): 723-729; doi: 10.1055/s-0042-104935.

25. *Bellia A, Iellamo F, De Carli E, Andreadi A, Padua E, Lombardo M, et al. Exercise individualized by TRIMPi method reduces arterial stiffness in early onset type 2 diabetic patients: a randomized controlled trial with aerobic interval training. Int J Cardiol. 2017;248:314319; doi: 10.1016/j.ijcard.2017.06.065.

26. *Cassidy SA, Thoma C, Hallsworth K, Jakovljevic DG, Parikh J, Hollingsworth KG, et al. High intensity intermittent exercise reverses abnormal cardiac function in people with type 2 diabetes: an MRI/S study. In: Boulton AJM, Del Prato S, Thorens B, Roden M, Walker M, Tack CJ, et al. (eds.), Minutes of the $49^{\text {th }}$ General Assembly of the European Association for the Study of Diabetes. Diabetologia. 2014;57(Suppl. 1):1566; S258. Available from: https://link.springer.com/ content/pdf/10.1007\%2Fs00125-014-3355-0.pdf.

27. *Cassidy S, Thoma C, Hallsworth K, Parikh J, Hollingsworth KG, Taylor R, et al. High intensity intermittent exercise improves cardiac structure and function and reduces liver fat in patients with type 2 diabetes: a randomised controlled trial. Diabetologia. 2016;59(1): 56-66; doi: 10.1007/s00125-015-3741-2.

28. *Cassidy S, Vaidya V, Houghton D, Zalewski P, Seferovic JP, Hallsworth K, et al. Unsupervised high-intensity interval training improves glycaemic control but not cardiovascular autonomic function in type 2 diabetes patients: a randomised controlled trial. Diab Vasc Dis Res. 2019;16(1):69-76; doi: 10.1177/14791 64118816223.

29. *Francois ME, Durrer C, Pistawka KJ, Halperin FA, Chang C, Little JP. Combined interval training and post-exercise nutrition in type 2 diabetes: a randomized control trial. Front Physiol. 2017;8:528; doi: 10.3389/ fphys.2017.00528.

30. *Hollekim-Strand SM, Bjørgaas MR, Albrektsen G, Tjønna AE, Wisløff U, Ingul CB. High-intensity interval exercise effectively improves cardiac function in patients with type 2 diabetes mellitus and diastolic dysfunction: a randomized controlled trial. J Am Coll Cardiol. 2014;64(16):1758-1760; doi: 10.1016/j.jacc. 2014.07.0971.

31. *Hua L, Lei M, Xue S, Li X, Li S, Xie Q. Effect of fish oil supplementation combined with high-intensity interval training in newly diagnosed non-obese type 2 diabetes: a randomized controlled trial. J Clin Biochem Nutr. 2020;66(2):146-151; doi: 10.3164/jcbn.19-64.

32. *Macías-Cervantes MH, Casillas LG, Sevilla MA, Figueroa A, Zarate E, Guerrero GA. Metabolic changes after two differents exercise programs in sedentary type 2 diabetic patients. Med Sci Sports Exerc. 2017; 49(5S):1020; doi: 10.1249/01.mss.0000519799.35679.86.

33. *Magalhães JP, Júdice PB, Ribeiro R, Andrade R, Raposo J, Dores H, et al. Effectiveness of high-intensity interval training combined with resistance training versus continuous moderate-intensity training com- 
bined with resistance training in patients with type 2 diabetes: a one-year randomized controlled trial. Diabetes Obes Metab. 2019;21(3):550-559; doi: 10.1111/ dom.13551.

34. *Mangiamarchi P, Caniuqueo A, Ramírez-Campillo R, Cárdenas P, Morales S, Cano-Montoya J, et al. Effects of high-intensity interval training and nutritional education in patients with type 2 diabetes [in Spanish]. Rev Med Chile. 2017;145(7):845-853; doi: 10.4067/ s0034-98872017000700845.

35. *Maillard F, Rousset S, Pereira B, Traore A, de Pradel Del Amaze P, Boirie Y, et al. High-intensity interval training reduces abdominal fat mass in postmenopausal women with type 2 diabetes. Diabetes Metab. 2016; 42(6):433-441; doi: 10.1016/j.diabet.2016.07.031.

36. *Mir E, Moazzami M, Bijeh N, Hakak Dokht E, Rahimi N. Changes in SFRP5, WNT5A, HbA1c, BMI, $\mathrm{PBF}$, and insulin resistance in men with type 2 diabetes after 12 weeks of combined exercise (HIIT and resistance). Int J Diabetes Dev Ctries. 2020;40(2):248-254; doi: 10.1007/s13410-019-00790-7.

37. *Mitranun W, Deerochanawong C, Tanaka H, Suksom D. Continuous vs interval training on glycemic control and macro- and microvascular reactivity in type 2 diabetic patients. Scand J Med Sci Sports. 2014; 24(2):e69-e76; doi: 10.1111/sms.12112.

38. *Støa EM, Meling S, Nyhus L-K, Strømstad G, Mangerud KM, Helgerud J, et al. High-intensity aerobic interval training improves aerobic fitness and $\mathrm{HbA1c}$ among persons diagnosed with type 2 diabetes. Eur J Appl Physiol. 2017;117(3):455-467; doi: 10.1007/ s00421-017-3540-1.

39. *Sudarsono NC, Tulaar ABM, Jusman SWA, Soewondo P, Sudaryo MDK, Siagian M, et al. The effects of combined high-intensity interval and resistance training on glycemic control and oxidative stress in T2DM. Asian J Sports Med. 2019;10(3):e91841; doi: 10.5812/asjsm.91841.

40. *Terada T, Friesen A, Chahal BS, Bell GJ, McCargar LJ, Boulé NG. Feasibility and preliminary efficacy of high intensity interval training in type 2 diabetes. Diabetes Res Clin Pract. 2013;99(2):120-129; doi: 10.1016/j. diabres.2012.10.019.

41. *Way KL, Sabag A, Sultana RN, Baker MK, Keating SE, Lanting S, et al. The effect of low-volume high-intensity interval training on cardiovascular health outcomes in type 2 diabetes: a randomised controlled trial. Int J Cardiol. 2020;320:148-154; doi: 10.1016/j.ijcard.2020. 06.019 .

42. *Wormgoor SG, Dalleck LC, Zinn C, Borotkanics R, Harris NK. High-intensity interval training is equivalent to moderate-intensity continuous training for shortand medium-term outcomes of glucose control, cardiometabolic risk, and microvascular complication markers in men with type 2 diabetes. Front Endocrinol. 2018; 9:475; doi: 10.3389/fendo.2018.00475.
43. American College of Sports Medicine. ACSM's guidelines for exercise testing and prescription, $10^{\text {th }} \mathrm{ed}$. Philadelphia: Wolters Kluwer; 2018.

44. Lora-Pozo I, Lucena-Anton D, Salazar A, Galán-Mercant A, Moral-Munoz JA. Anthropometric, cardiopulmonary and metabolic benefits of the high-intensity interval training versus moderate, low-intensity or control for type 2 diabetes: systematic review and metaanalysis. Int J Environ Res Public Health. 2019;16(22): 4524; doi: 10.3390/ijerph16224524.

45. Afrasyabi S, Marandi SM, Kargarfard M. The effects of high intensity interval training on appetite management in individuals with type 2 diabetes: influenced by participants weight. J Diabetes Metab Disord. 2019; 18(1):107-117; doi: 10.1007/s40200-019-00396-0.

46. Mottalib A, Salsberg V, Mohd-Yusof B-N, Mohamed W, Carolan P, Pober DM, et al. Effects of nutrition therapy on HbA1c and cardiovascular disease risk factors in overweight and obese patients with type 2 diabetes. Nutr J. 2018;17(1):42; doi: 10.1186/s12937-018-0351-0.

47. Peng XV, Ayyagari R, Lubwama R, Shi L, Price-Haywood EG, Hollander P, et al. Impact of simultaneous versus sequential initiation of basal insulin and glucagon-like peptide-1 receptor agonists on HbA1c in type 2 diabetes: a retrospective observational study. Diabetes Ther. 2020;11(4):995-1005; doi: 10.1007/s13300020-00783-4.

48. Abusaib M, Ahmed M, Nwayyir HA, Alidrisi HA, AlAbbood M, Al-Bayati A, et al. Iraqi experts consensus on the management of type 2 diabetes/prediabetes in adults. Clin Med Insights Endocrinol Diabetes. 2020; 13:1-11; doi: 10.1177/1179551420942232.

49. Devitskaya D, Villalobos C, VanNess JM, Vosti PD, King AC, Jensen CD. Sex-specific HbA1c responses to structured exercise among patients with type 2 diabetes. Med Sci Sports Exerc. 2019;51(6S):299-300; doi: 10.1249/01.mss.0000561405.06744.80.

50. Montes de Oca García A, Gutiérrez Manzanedo J, Ponce González JG. High-intensity interval training (HIIT) as a therapeutic tool in patients with type 2 diabetes mellitus: a narrative review [in Spanish]. Retos. 2019; 36(2):633-639; doi: 10.47197/retos.v36i36.69762. 